\title{
(6) OPEN ACCESS \\ Adding tocilizumab or switching to tocilizumab monotherapy in methotrexate inadequate responders: 24-week symptomatic and structural results of a 2-year randomised controlled strategy trial in rheumatoid arthritis (ACT-RAY)
}

\author{
Maxime Dougados, ${ }^{1}$ Karsten Kissel, ${ }^{2}$ Tom Sheeran, ${ }^{3}$ Paul P Tak, ${ }^{4}$ Philip G Conaghan, ${ }^{5}$ \\ Emilio Martín Mola, ${ }^{6}$ Georg Schett, ${ }^{7}$ Howard Amital, ${ }^{8}$ Federico Navarro-Sarabia, ${ }^{9}$ \\ Antony Hou, ${ }^{10}$ Corrado Bernasconi, ${ }^{2}$ TWJ Huizinga ${ }^{11}$
}

\begin{abstract}
- Additional supplementary data are published online only. To view the files please visit the journal online (http:/dx.doi.org/ 10.1136/annrheumdis-2011201282).
\end{abstract}

1Department of Rheumatology, Cochin Hospital, Paris, France ${ }^{2} \mathrm{~F}$ Hoffmann-La Roche Ltd, Basel, Switzerland

${ }^{3}$ Cannock Rheumatology Centre, Cannock, UK

${ }^{4}$ Department of Clinical Immunology and Rheumatology, AMC University of Amsterdam, Amsterdam, The Netherlands (GlaxoSmithKline since Jan 2011) ${ }^{5}$ Section of Musculoskeletal Disease, University of Leeds and NIHR Leeds Musculoskeletal Biomedical Research Unit, Leeds, UK

${ }^{6}$ Department of Rheumatology, Universidad Autónoma de Madrid, Madrid, Spain

7Department of Rheumatology, University of Erlangen-

Nuremberg, Erlangen, Germany ${ }^{8}$ Department of Medicine, Sheba Medical Center Tel-Hashomer, Israel ${ }^{9}$ Rheumatology Department, Hospital Universitario Virgen Macarena, Sevilla, Spain

${ }^{10}$ Inland Rheumatology Clinical Trials, Upland, California, USA

${ }^{11}$ Department of Rheumatology, Leiden University Medical Center, Leiden, The Netherlands

\section{Correspondence to}

Maxime Dougados,

Department of Rheumatology, APHP, Cochin Hospital, 27 Rue du Faubourg Saint-Jacques, 75014 Paris, France: maxime.dougados@cch.aphp.fr

Accepted 7 March 2012

Published Online First

7 July 2012

\section{ABSTRACT}

Objective In patients with active rheumatoid arthritis (RA) despite methotrexate, to compare the efficacy of adding tocilizumab to that of switching to tocilizumab monotherapy.

Methods Double-blind, 2-year study in which adults with active RA (DAS28 > 4.4) despite methotrexate were randomly assigned either to continue methotrexate with the addition of tocilizumab (MTX+TCZ) $8 \mathrm{mg} / \mathrm{kg}$ every 4 weeks or switch to tocilizumab and placebo (TCZ+PBO). The primary endpoint was the DAS28-erythrocyte sedimentation rate (ESR) remission rate at week 24 . Secondary objectives included other symptomatic outcomes, quality of life and progression of structural damage.

Results Of 556 randomly assigned patients, 512 (92\%) completed 24 weeks. DAS28-ESR remission rates were 40.4\% for TCZ+MTX and 34.8\% for TCZ+PBO $(p=0.19)$; American College of Rheumatology 20/50/70/90 rates were $71.5 \% / 45.5 \% / 24.5 \% / 5.8 \%$ (TCZ + MTX) and 70.3\%/40.2\%/25.4\%/5.1\% (TCZ+PBO; differences not significant). A significant difference between groups was seen for low DAS28 (61.7\% vs 51.4\%). Radiographic progression was small and not different between groups (Genant-Sharp score progression $\leq$ smallest detectable change in 91\% (TCZ+MTX) and 87\% (TCZ+PBO)). Rates per 100 patient-years of serious adverse events and serious infections were 21 and six, respectively, for TCZ+MTX and 18 and six, respectively, for TCZ+PBO. Alanine aminotransferase elevations greater than threefold the upper limit of normal occurred in $7.8 \%$ and $1.2 \%$ of TCZ+MTX and TCZ+PBO patients, respectively. Conclusion No clinically relevant superiority of the TCZ+MTX add-on strategy over the switch to tocilizumab monotherapy strategy was observed. The combination was more commonly associated with transaminase increases. Meaningful clinical and radiographic responses were achieved with both strategies, suggesting that tocilizumab monotherapy might be a valuable treatment strategy in suitable RA patients.

One of the major long-term objectives of rheumatoid arthritis (RA) treatment is to prevent functional impairment as a result of bone damage, tendon or ligament rupture and cartilage breakdown.
Persistent inflammation at the level of the joint (synovitis and osteitis) or the whole body (reflected in acute phase reactants) is among the most important predictors of subsequent structural deterioration. ${ }^{1}$ Inflammation is also responsible for symptoms such as pain, fatigue and disability that impair the patient's quality of life. ${ }^{2}$ Structural deterioration can be evaluated over months using radiological scoring systems. ${ }^{3}$ Therefore, the short-term objective of RA treatment is to improve the patient's condition by abrogating inflammation and by sustaining this, thereby achieving the longer term objective of stopping radiological progression. ${ }^{1}$

Methotrexate is considered the cornerstone of therapy to achieve this goal. When there is inadequate disease control with methotrexate alone, the current recommendation is to add a tumour necrosis factor blocker or another approved biological agent. ${ }^{4}$ However, as evidenced by registries of routine clinical practice treatment, approximately one third of RA patients are being treated with biological monotherapy, that is without concomitant methotrexate. ${ }^{5} 6$ There are many reasons for stopping methotrexate or initiating biological agents as a monotherapy. In daily practice, frequent methotrexate-induced gastrointestinal disorders (eg, nausea) have been reported as leading to poor patient compliance. ${ }^{7}$ Moreover, the use of methotrexate may lead to other safety issues such as haematological and hepatic adverse events. Such limitations explain why it is important to evaluate a switch strategy to biological monotherapy in addition to traditional add-on strategies (ie, the addition of a biological agent to methotrexate).

Tocilizumab, a humanised antihuman interleukin-6 receptor monoclonal antibody ${ }^{8}$ has proved its efficacy and safety in RA patients continuing to receive methotrexate ${ }^{9} 10$ and as biological monotherapy. ${ }^{11}$ The latter is supported by data from a head-to-head trial showing that tocilizumab was more efficacious than methotrexate in patients who had not failed previous treatment with methotrexate or biological agents. ${ }^{12}$ Because methotrexate is the current recommended first-line therapy, the question arises as to whether tocilizumab should 
be added to methotrexate (add-on strategy) or methotrexate could be stopped when commencing tocilizumab (switch strategy) in patients with inadequately controlled disease. The only data comparing the two strategies is from a phase II study with a small sample size and no structural outcome measures to indicate the superiority of the add-on strategy. ${ }^{13}$

We therefore conducted a 2-year trial with the objective of assessing the efficacy and safety profile of either adding tocilizumab to methotrexate or switching methotrexate to tocilizumab monotherapy in patients with persistent active disease despite methotrexate therapy. Here, we report the first 24 -week clinical and radiological data.

\section{PATIENTS AND METHODS}

Study design

This report covers the planned analysis of the first 24 weeks (including the primary endpoint) of an on-going 2-year double-blind placebo controlled parallel-group clinical trial (NCT00810199, EudraCT no 2008-001847-20). The treatment allocation of individual patients remained blinded for patients, site personnel and the data analysis/interpretation team, except for the separate subgroup technically preparing the data.

The study was approved by the appropriate institutional review boards/ethics committees with written informed consent obtained from each patient before study participation. The study was conducted in full accordance with International Conference on Harmonisation/good clinical practice and the principles, laws and regulations of the countries in which the research was conducted.

\section{Patients}

Eligible patients had confirmed RA according to the 1987 American College of Rheumatology (ACR) criteria with active disease defined as disease activity score based on 28 jointserythrocyte sedimentation rate (DAS28-ESR) greater than 4.4 at baseline and 4.0 or more at screening, and had been receiving methotrexate for at least 12 weeks, with a stable dose of at least $15 \mathrm{mg} /$ week for 6 weeks or longer before starting study treatment. For inclusion, patients were also required to have bone damage with radiographic evidence of at least one joint with definite erosion attributable to RA as determined by a central reader. Major exclusion criteria included severe comorbidities, any previous use of biological agents as well as any conventional disease-modifying antirheumatic drug treatment other than methotrexate during the month ( 3 months for leflunomide) preceding the baseline visit (see supplementary data, available online only, for full inclusion and exclusion criteria).

\section{Study treatment}

Patients were randomly assigned either to the add-on or the switch strategy group (see supplementary data, available online only, for study design schematic). Randomisation was stratified by study site and baseline DAS28-ESR ( $\leq$ or $>5.5$ ) using a minimisation algorithm. All patients received open-label tocilizumab $8 \mathrm{mg} / \mathrm{kg}$ intravenously every 4 weeks. Treatment with methotrexate/placebo was double-blind: all patients received identical capsules of either placebo (switch strategy arm) or methotrexate $2.5 \mathrm{mg}$ (add-on strategy arm), with the number of capsules at study entry being consistent with prestudy dosage. Tocilizumab and/or disease-modifying antirheumatic drug treatment was reduced or temporarily interrupted in patients with alanine aminotransferase or aspartate transaminase values greater than one to three times the upper limit of normal (ULN), and was discontinued for persistent increases greater than three times ULN.

\section{Concomitant RA treatments}

Oral corticosteroids ( $\leq 10 \mathrm{mg} /$ day prednisone or equivalent) and non-steroidal anti-inflammatory drugs were permitted as long as doses had been stable for at least 25 of 28 days before the start of study treatment. Alterations in the non-steroidal anti-inflammatory drug dose were not recommended during the study, particularly during the first 24 weeks. The corticosteroid dose could not be changed during the first 24 weeks of the study.

\section{Collected patient data and assessments}

Data collected at baseline included demographics and disease characteristics such as RA disease duration. At baseline and every 4 weeks thereafter the following parameters were assessed: tender joint count, swollen joint count, health assessment questionnaire-disability index (HAQ-DI), patient's global assessment, physician's global assessment, C-reactive protein (CRP), ESR. Quality of life was assessed at baseline and at weeks 4, 8, 12 and 24 using the rheumatoid arthritis quality of life questionnaire (RAQoL). At each visit, patients were monitored for adverse events, vital signs and laboratory tests (eg, blood counts, transaminases, cholesterol). Radiographs of the hands/ wrists and feet were obtained at baseline and week 24. Each radiograph was assessed applying the Genant-modified Sharp scoring system (GSS) by two independent readers (Perceptive Informatics Medical Imaging Services, Berlin, Germany) who were blinded to treatment assignment, chronological order of radiographs and patient's clinical status. The smallest detectable change (SDC) for GSS was computed based on the observed SD of difference between the x-ray readers, ${ }^{14}$ whereas three readers in total participated in the campaign. The SDC is the smallest change that can be attributed to something more than observed variability of reader differences.

\section{Statistical analysis}

The clinical superiority of the tocilizumab plus methotrexate combination treatment in the phase II CHARISMA study ${ }^{13}$ had an important influence on the statistical parts of the design.

To assess the working hypothesis that the add-on strategy (ie, initiation of tocilizumab plus continuation of methotrexate) would be superior to the switch strategy (ie, initiation of tocilizumab and discontinuation of methotrexate), the primary outcome measure of the study was defined as the percentage of patients in remission according to DAS28-ESR (DAS28 <2.6) at week 24. A two-sided statistical test of no difference between the two treatment arms at the 5\% significance level was used. Specifically for the primary endpoint, as well as for similar endpoints, a logistic regression model was employed including the stratification factors used at randomisation (site and baseline DAS28 $\leq$ or $>5.5$ ) with a supportive Cochran-Mantel-Haenszel test stratified for the same parameters. Analysis of covariance models were used to compare continuous outcome measures.

Efficacy analyses were conducted in the intention-to-treat population (all randomly assigned and treated patients analysed in the arm they were randomly assigned to) with non-responder imputation for categorical variables (eg, DAS28 remission, ACR response), last observation carried forward until patient withdrawal for missing joint counts and no additional imputation of missing values.

The target sample size (235 patients per arm) was computed to provide $80 \%$ power to detect a $12.5 \%$ treatment effect 


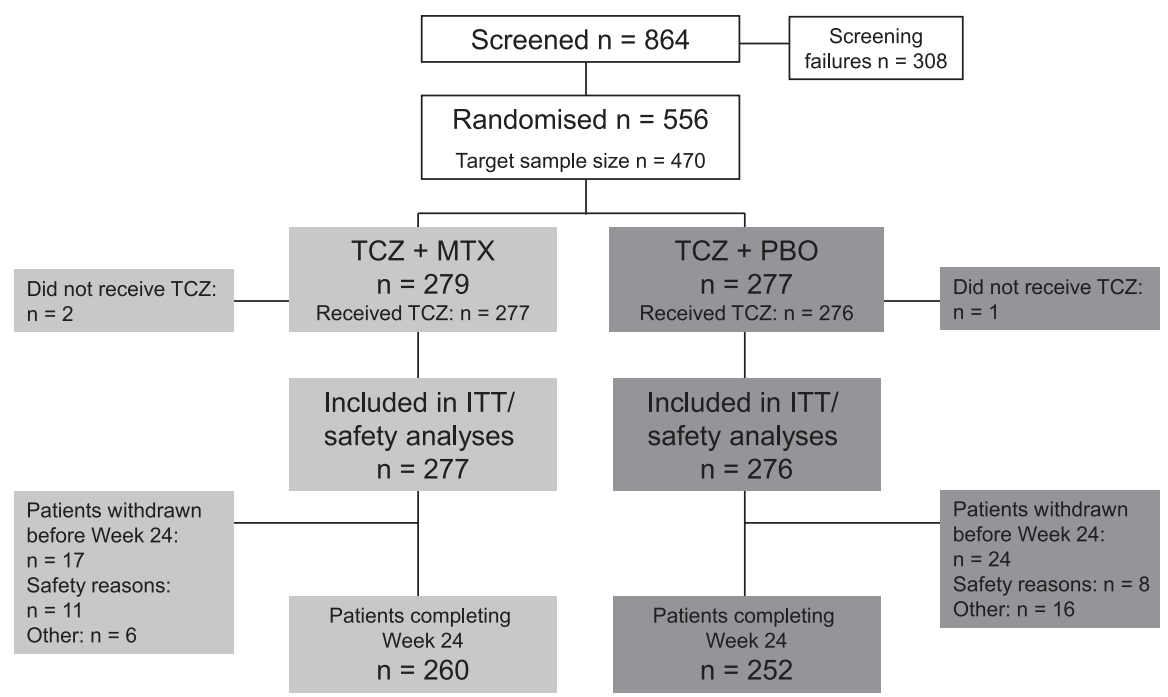

Figure 1 Patient disposition and study flow chart. ITT, intention-to-treat; MTX, methotrexate; PBO, placebo; TCZ, tocilizumab.

difference between an expected $42.5 \%$ DAS28 remission rate at week 24 in the add-on strategy arm versus $30 \%$ in the switch strategy arm. ${ }^{12}$ This difference $(12.5 \%)$ was deemed to be clinically relevant by the study's steering committee (see supplementary data, available online only).

The following additional endpoints were analysed in accordance with the European League Against Rheumatism (EULAR)/ ACR collaborative recommendations for reporting RA disease activity in clinical trials: ${ }^{11}$

(1) Other outcome measures at week 24 included mean changes in DAS28-ESR and in selected variables (eg, swollen joint count, tender joint count, ESR, CRP, HAQ-DI, RAQoL); percentage of patients who improved during the 24 weeks of the study according to ACR20/50/70/90, EULAR response and the percentage of patients with DAS28 <3.2 (low disease activity state). Remission as defined by the 2010 ACR-EULAR criteria (Boolean definition), ${ }^{15}$ simplified disease activity index $\leq 3.3$ and clinical disease activity index $\leq 2.8$ were analysed post hoc.

(2) To approach the concepts of onset of action and sustainability, changes over time of selected variables such as joint counts, CRP, pain and HAQ-DI were evaluated.

(3) The domain fatigue was evaluated using question 21 of the RAQoL questionnaire.

(4) Radiographic endpoints included changes from baseline in total GSS, erosion and joint space narrowing scores and the proportion of patients with no radiographic progression (progression defined as change in GSS $>$ SDC or $>0$ ).

Safety endpoints included the incidence of adverse events $(\mathrm{AE})$, serious $\mathrm{AE}$, serious infections and specific laboratory abnormalities, which were analysed in the safety population (all treated patients with at least one post-dose assessment of safety, analysed according to the treatment received).

\section{RESULTS}

\section{Patient flow and baseline characteristics}

Figure 1 summarises the flow of patients. The predominant reason for screening failure $(n=308)$ was the absence of radiological erosions ( $40 \%$ of screening failures). Five hundred and fifty-six patients were recruited, exceeding the target of 470 patients. This larger sample size increased the precision of the estimates.
Table 1 Baseline characteristics

\begin{tabular}{|c|c|c|}
\hline & $\begin{array}{l}\text { TCZ+MTX } \\
(\mathrm{N}=277)\end{array}$ & $\begin{array}{l}\mathrm{TCZ}+\mathrm{PBO} \\
(\mathrm{N}=276)\end{array}$ \\
\hline Women, n (\%) & $227(81.9)$ & $217(78.6)$ \\
\hline Age (years), mean (SD) & $53.0(13.4)$ & $53.6(11.9)$ \\
\hline Patients aged $\geq 65$ years, $n(\%)$ & $53(19.1)$ & $52(18.8)$ \\
\hline $\mathrm{BMI}, \mathrm{kg} / \mathrm{m}^{2}$, mean (SD) & $26.3(5.20)$ & $26.5(5.14)$ \\
\hline Duration of RA, years, mean (SD) & $8.2(8.0)$ & $8.3(8.4)$ \\
\hline \multicolumn{3}{|l|}{$\begin{array}{l}\text { Categorical duration of RA in years, } \\
n(\%) \text { : }\end{array}$} \\
\hline$<2$ & $51(18.4)$ & $66(23.9)$ \\
\hline$\geq 2$ to $<5$ & 76 (27.4) & $68(24.6)$ \\
\hline$\geq 6$ to $<10$ & $66(23.8)$ & $63(22.8)$ \\
\hline$\geq 10$ & $84(30.3)$ & $79(28.6)$ \\
\hline Swollen joint count, mean (SD) & $14.4(8.9)$ & $15.3(10.2)$ \\
\hline Tender joint count, mean (SD) & $25.8(13.9)$ & $26.6(15.2)$ \\
\hline DAS28-ESR, mean (SD) & $6.33(0.98)$ & $6.36(1.00)$ \\
\hline HAQ-DI, mean (SD) & $1.46(0.66)$ & $1.48(0.60)$ \\
\hline $\mathrm{HAQ}-\mathrm{DI}<0.5, \mathrm{n}(\%)$ & $17(6.2)$ & $14(5.2)$ \\
\hline Genant-modified Sharp score, mean (SD) & $30.4(31.8)$ & $37.1(40.5)$ \\
\hline Methotrexate dose, mg/week, mean (SD) & $16.0(4.4)$ & $16.2(4.1)$ \\
\hline Methotrexate dose, mg/week, median & 15.0 & 15.0 \\
\hline $\begin{array}{l}\text { No of previous DMARD (including methotrexate } \\
\text { before study entry), mean (SD) }\end{array}$ & $1.9(1.1)$ & $1.9(1.0)$ \\
\hline Oral steroid use, $\mathrm{n}(\%)$ & $136(49.1)$ & $135(48.9)$ \\
\hline Folic acid use, n (\%) & $215(77.6)$ & $224(81.2)$ \\
\hline
\end{tabular}

BMI, body mass index; DMARD, disease-modifying antirheumatic drug; DAS28-ESR, disease activity score based on 28 joints-erythrocyte sedimentation rate; $\mathrm{HAO}-\mathrm{DI}$, health assessment questionnaire-disability index; MTX, methotrexate; PBO, placebo; $\mathrm{RA}$, rheumatoid arthritis; TCZ, tocilizumab.

Of the 556 randomly assigned patients, 512 (92\%) completed the first 24 weeks of the trial. There were no clinically significant differences between the groups in the baseline characteristics except for a potentially relevant difference in GSS (table 1).

\section{Clinical efficacy}

The main efficacy results at week 24 are summarised in figure 2 and table 2 (also see supplementary data, available online only). Patients received mean weekly doses of methotrexate/placebo ranging from 15.2 to $15.9 \mathrm{mg} /$ week and 15.8 to $16.3 \mathrm{mg} /$ week 
TCZ + MTX $(n=277) \quad \square T C Z+P B O(n=276)$

A
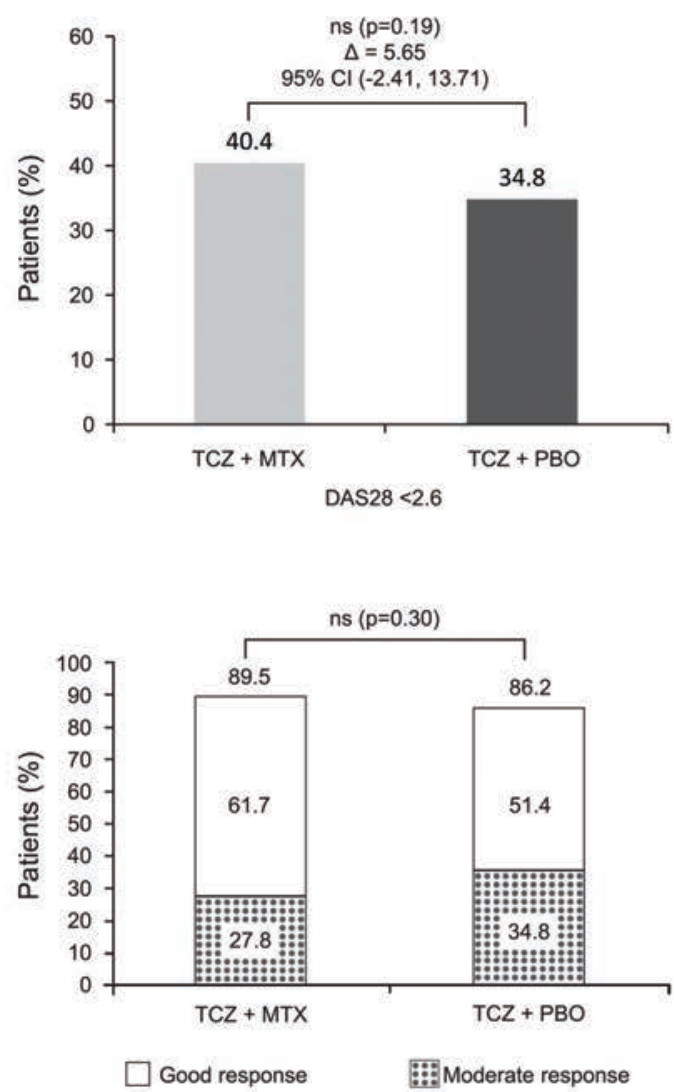

$E$

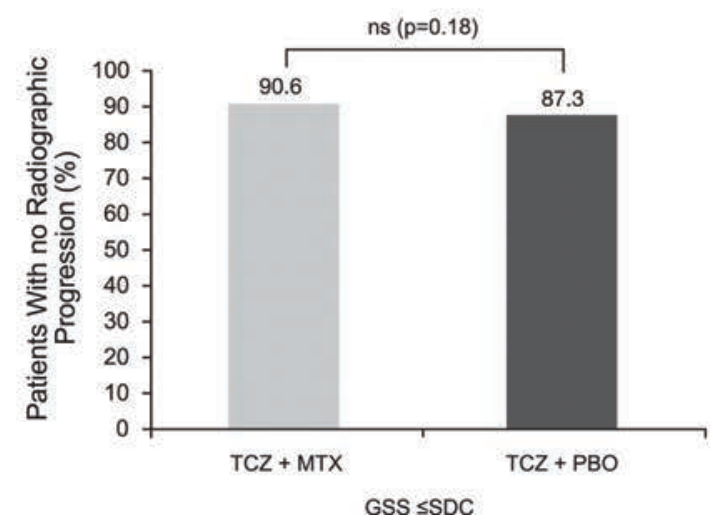

B

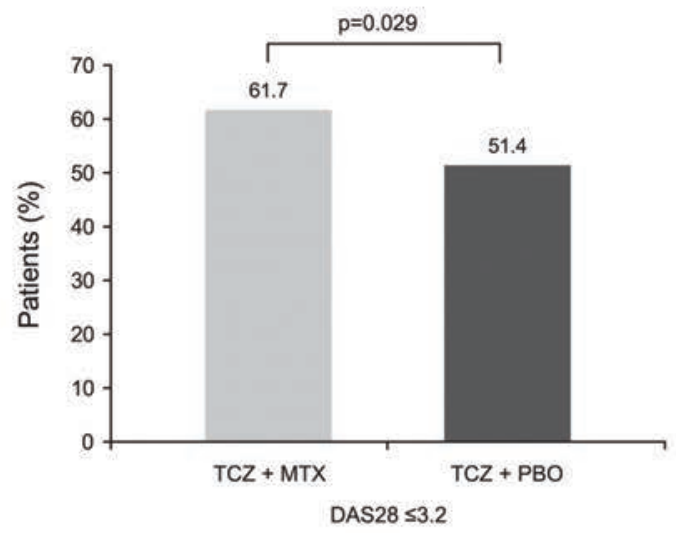

D

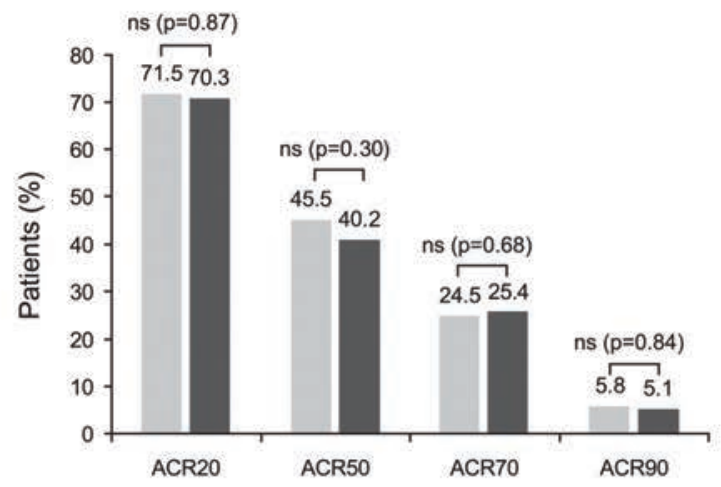

F

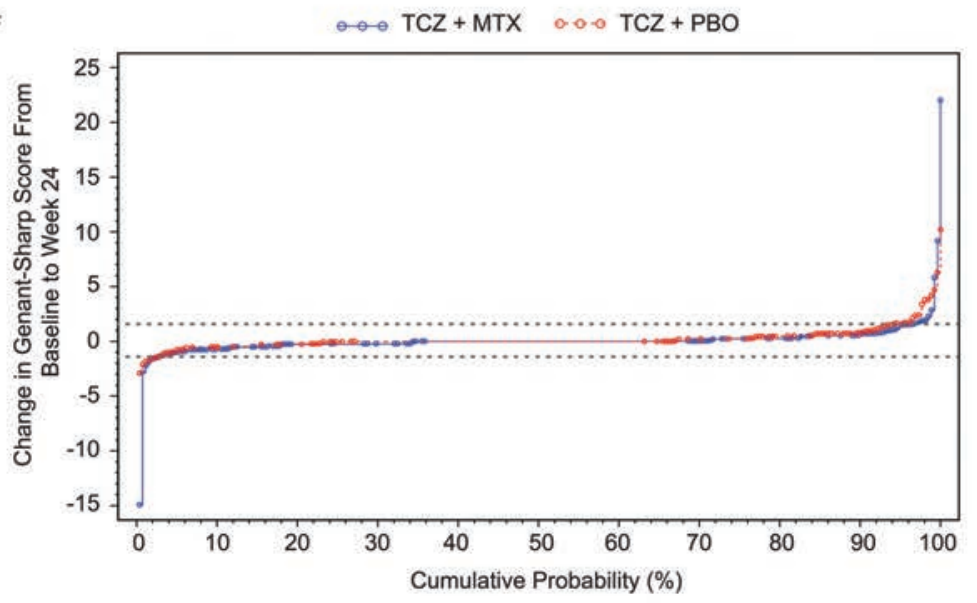

Figure 2 Binary composite indices and radiographic results at week 24 (intent-to-treat population). (A) Patients achieving remission (DAS28 <2.6), (B) low disease activity (DAS28 $\leq 3.2$ ), (C) good or moderate European League Against Rheumatism responses, (D) ACR responses, (E) patients (\%) with no radiographic progression in total GSS, and $(F)$ cumulative distribution of change from baseline in total GSS. Dashed lines indicate smallest detectable change with data falling between these lines representing no change. ACR, American College of Rheumatology; DAS28, disease activity score based on 28 joints; GSS, Genant-modified Sharp Score; MTX, methotrexate; NS, not significant; PBO, placebo; TCZ, tocilizumab.

for the tocilizumab plus methotrexate and tocilizumab plus placebo groups, respectively. Both treatment strategies showed a highly relevant clinical treatment effect. The study did not demonstrate a statistically significant difference between the two groups in DAS28-ESR remission rates (primary endpoint). The actual absolute difference between groups $(5.65 \%, 95 \% \mathrm{CI}$
$-2.41 \%$ to $13.71 \%, p=0.19)$ was much smaller than what had been considered clinically relevant a priori $(12.5 \%)$. For other endpoints, the differences between the treatment groups at week 24 were mostly not statistically significant and/or small, but with a numerical trend towards superiority of the add-on strategy. The difference between groups in the proportion of 
Table 2 Efficacy results at week 24

\begin{tabular}{|c|c|c|c|}
\hline Variable & $\mathrm{TCZ}+\mathrm{MTX}(\mathrm{N}=277)$ & $\mathrm{TCZ}+\mathrm{PBO}(\mathrm{N}=276)$ & $\begin{array}{l}\text { p Value } \\
\text { (between group) }\end{array}$ \\
\hline DAS28 remission rate, $\%$ & 40.4 & 34.8 & 0.19 \\
\hline Change in DAS28, mean (SD) & $-3.43(1.33)$ & $-3.21(1.31)$ & 0.051 \\
\hline LDAS, \% & 61.7 & 51.4 & 0.029 \\
\hline EULAR good plus moderate responders, $\%$ & 89.5 & 86.2 & 0.30 \\
\hline ACR-EULAR Boolean remission rate, $\%$ & 6.9 & 5.4 & 0.53 \\
\hline SDAl remission rate $(\leq 3.3), \%$ & 11.9 & 9.8 & 0.56 \\
\hline CDAl remission rate $(\leq 2.8), \%$ & 11.9 & 7.6 & 0.12 \\
\hline Change in tender joint count, mean (SD) & $-17.25(13.35)$ & $-17.00(13.64)$ & 0.52 \\
\hline Change in swollen joint count, mean (SD) & $-11.33(8.04)$ & $-11.75(9.45)$ & 0.61 \\
\hline Change in patient's global assessment of disease activity, mean (SD) & $-34.3(25.68)$ & $-32.4(24.34)$ & 0.20 \\
\hline Change in physician's global assessment of disease activity, mean (SD) & $-40.7(19.55)$ & $-38.5(21.65)$ & 0.084 \\
\hline Change in patient's global assessment of pain, mean (SD) & $-29.3(26.64)$ & $-29.8(24.92)$ & 0.30 \\
\hline Change in $\mathrm{RAOOL}$, mean (SD) & $-5.97(7.95)$ & $-5.19(7.06)$ & $0.31^{*}$ \\
\hline Change in total GSS, mean (SD) & $0.08(1.88)$ & $0.22(1.11)$ & 0.26 \\
\hline Change in JSN score, mean (SD) & $0.08(1.48)$ & $0.11(0.70)$ & 0.76 \\
\hline Change in erosion score, mean (SD) & $-0.01(0.78)$ & $0.11(0.63)$ & 0.066 \\
\hline Patients with no progression in GSS ( $\leq \mathrm{SDC}), \%$ & 90.6 & 87.3 & 0.18 \\
\hline Patients with no progression in GSS $(\leq 0), \%$ & 65.7 & 59.1 & 0.088 \\
\hline
\end{tabular}

ACR, American College of Rheumatology; CDAl, clinical disease activity index; DMARD, disease-modifying antirheumatic drug; DAS28, disease activity score based on 28 joints; EULAR, European League Against Rheumatism; GSS, Genant-modified Sharp score; JSN, joint space narrowing; LDAS, low disease activity score; MTX, methotrexate; PBO, placebo; RAQoL, rheumatoid arthritis quality of life questionnaire; SDAl, simplified disease activity index; SDC, smallest detectable change; TCZ, tocilizumab. *p Value from a 2-sided Wilcoxon rank-sum test of no difference between the two treatment arms.

patients achieving low disease activity (DAS28-ESR <3.2) was $10.3 \%(p=0.029)$.

Fatigue, as assessed by the proportion of patients answering 'yes' to question 21 of the RAQoL questionnaire ('I feel tired whatever I do') affected $75.6 \%$ and $73.3 \%$ of patients at baseline (add-on vs switch, respectively, $\mathrm{p}=0.79$ ) and $51.9 \%$ and $50.0 \%$ at week $24(p=0.68)$. Clinical parameters and CRP improved rapidly in both groups (figure $3 \mathrm{~A}-\mathrm{D}$ ). For all these variables a sustained or continuously increasing effect size was observed in both treatment groups through the first 24 weeks of the study.

\section{Progression of structural damage}

There were no statistically significant intergroup differences in any of the evaluated continuous outcome measures (table 2, figure 3E). The SDC from baseline in GSS was 1.5, indicating a high agreement of the readings, therefore allowing for the detection of changes from baseline less than -1.5 and greater than 1.5. Radiographic progression was defined in two ways: any change in GSS greater than zero or greater than SDC. Based on the second definition the proportion of patients with no radiographic progression was $87.3 \%$ and $90.6 \%$ (GSS $\leq \mathrm{SDC}$ ) in the tocilizumab plus placebo and tocilizumab plus methotrexate groups, respectively, and was generally very low in those patients who still had progressive structural damage. The changes from baseline in total GSS at week 24 were distributed similarly in the two groups (figure $2 \mathrm{~F}$ ).

\section{Safety}

A safety overview is presented in table 3. Overall rates per 100 patient-years of $\mathrm{AE}$ and serious $\mathrm{AE}$ were similar between groups. A total of 17 patients (6.1\%) in the tocilizumab plus methotrexate group and 16 patients $(5.8 \%)$ in the tocilizumab plus placebo group experienced serious $\mathrm{AE}$, the most common of which were infections (seven events in each group) and cardiac disorders (three and four events in the two groups, respectively). There was a total of three deaths in the first 24 weeks and a fourth patient died from an $\mathrm{AE}$ that started within the first 24 weeks (two each in either group). The causes of death in these four patients were: sepsis; septic shock preceded by scrotal abscess, skin necrosis, acute renal failure and congestive heart failure; myocardial infarction; and sepsis with meningitis. AE-related discontinuations and dose modifications occurred in $3.6 \%$ and $27.4 \%$ of tocilizumab plus methotrexate patients and $2.5 \%$ and $18.5 \%$ of tocilizumab plus placebo patients, respectively.

Alanine aminotransferase elevations from normal at baseline to greater than ULN and to more than three times ULN at one or more time points during 24 weeks occurred in $48.8 \%$ and $7.8 \%$ of tocilizumab plus methotrexate and in $27.6 \%$ and $1.2 \%$ of tocilizumab plus placebo patients, respectively. For aspartate transaminase, such elevations occurred in $34.2 \%$ and $1.9 \%$ (tocilizumab plus methotrexate) and in $19.2 \%$ and $0.4 \%$ (tocilizumab plus placebo) of patients, respectively, indicating a trend towards lower rates of liver enzyme elevations with tocilizumab monotherapy. As seen in previous studies with tocilizumab and also with other anti-inflammatory treatments, mean total cholesterol levels increased from 93.1 and $92.7 \mathrm{mg} / \mathrm{dl}$ at baseline to 102.4 and $103.5 \mathrm{mg} / \mathrm{dl}$ at week 24 in the tocilizumab plus methotrexate and tocilizumab plus placebo groups, respectively.

\section{DISCUSSION}

This study evaluated two different tocilizumab-based treatment strategies in patients with active RA and suggests that a clinically relevant effect with arrest of structural progression is observed at 24 weeks with both treatment strategies in the majority of patients. The study did not succeed at demonstrating that addon strategy efficacy (combination therapy of tocilizumab plus methotrexate) was superior to the switch strategy (monotherapy tocilizumab plus placebo), although there were numerically small and not clinically meaningful differences in the primary and some secondary efficacy endpoints in favour of combination therapy. No differences were observed in safety, the exception being that the add-on strategy resulted in a numerically higher percentage of patients who had transaminase level increases compared with the switch strategy. 
$-\mathrm{TCZ}+\mathrm{MTX}$

A

Swollen Joint Count

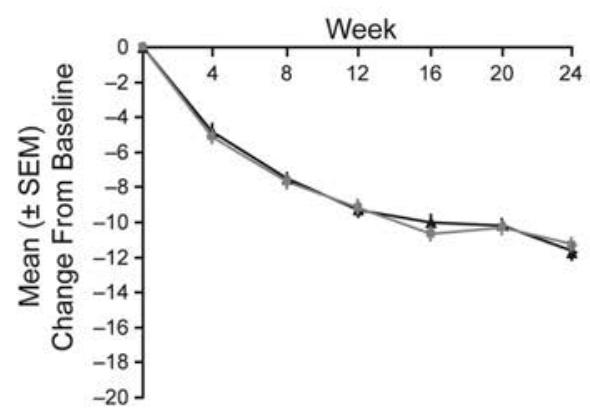

C

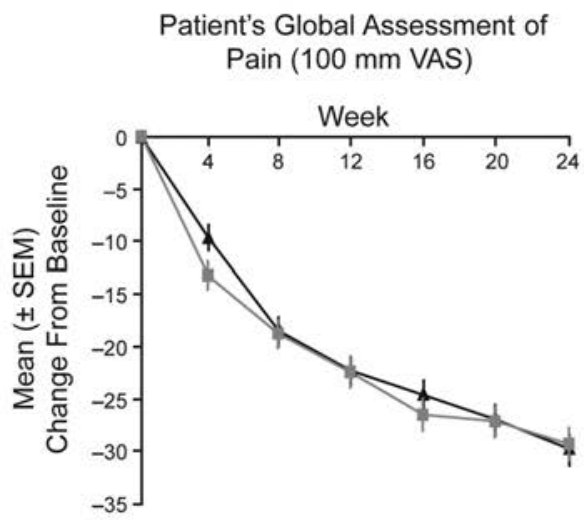

E

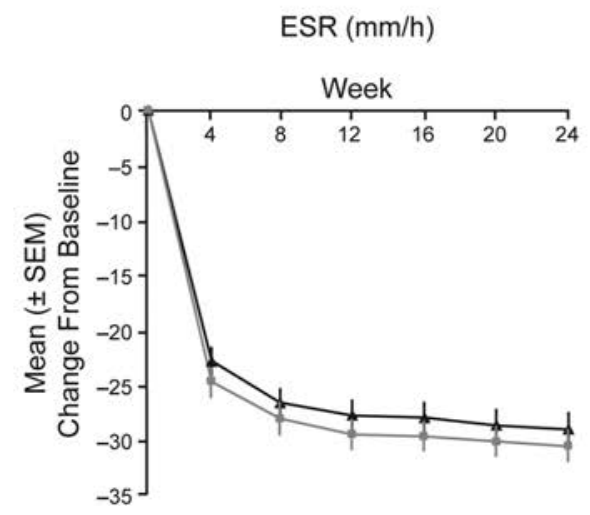

$\mathrm{TCZ}+\mathrm{PBO}$

B

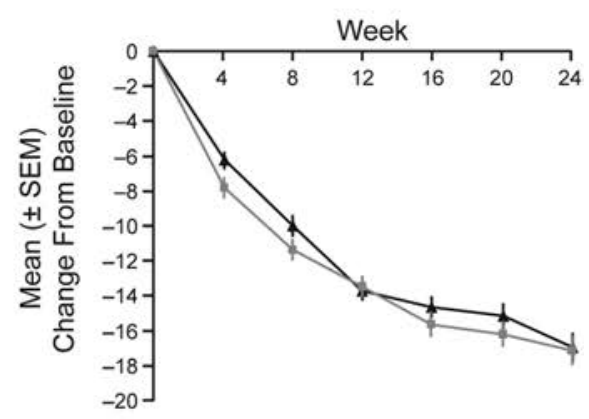

D

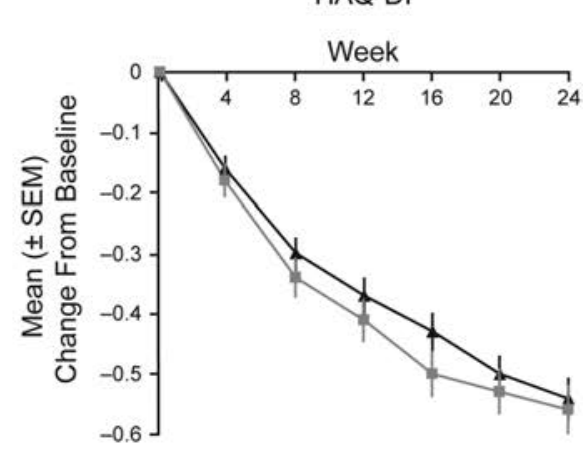

F

CRP $(\mathrm{mg} / \mathrm{ml})$

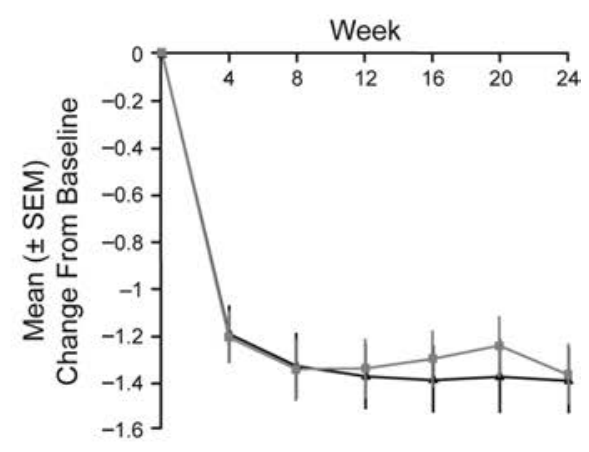

Figure 3 Changes from baseline in selected American College of Rheumatology core set variables over time (intent-to-treat population). (A) Mean change from baseline for swollen joint counts, (B) tender joint counts, (C) patient's global assessment of pain, (D) HAQ-DI, (E) ESR and (F) CRP. CRP, C-reactive protein; ESR, erythrocyte sedimentation rate; HAQ-DI, health assessment questionnaire-disability index; MTX methotrexate; TCZ, tocilizumab; VAS, visual analogue scale.

This is the first study comparing the efficacy and safety of tocilizumab in combination with methotrexate and as monotherapy in inadequate responders to methotrexate with a sufficient sample size to address this question prospectively. The primary outcome measure (DAS28-ESR remission rate at 24 weeks) did not differ significantly between the two strategies. With the exception of the proportion of patients with low disease activity (DAS28-ESR <3.2) at 24 weeks, the secondary efficacy analyses supported the primary endpoint by not showing a between-group statistically significant difference. This interpretation is also supported by the changes over time in individual parameters (figure 2). Looking at the overall picture across different endpoints and time points there was a trend towards slightly higher responses with the add-on strategy (eg, 5.6\% difference in DAS28 remission and 3.3\% difference in patients with no radiological progression greater than SDC). Looking at the effect sizes and the fact that most of the differences are not statistically significant the trend in favour of the add-on strategy is considered clinically not meaningful.

As the study did not succeed at demonstrating the superiority of the add-on versus the switch strategy, we discuss here factors that could potentially have biased the study outcome, before 
Table 3 Overview of adverse events, deaths, and liver enzyme elevations* ${ }^{*}$ until week 24

\begin{tabular}{|c|c|c|}
\hline & TCZ+MTX & $\mathrm{TCZ}+\mathrm{PBO}$ \\
\hline & $\mathrm{N}=\mathbf{2 7 7}$ & $\mathrm{N}=\mathbf{2 7 6}$ \\
\hline Total tocilizumab exposure (PY) & 118.31 & 116.40 \\
\hline \multicolumn{3}{|l|}{$\mathrm{AE}$} \\
\hline Total patients with $\geq 1 \mathrm{AE}, \%$ (n) & $70.0 \%(194)$ & $72.5 \%(200)$ \\
\hline Total no of AE & 581 & 544 \\
\hline Rate of $\mathrm{AE}$ (per $100 \mathrm{PY}$ ) & 491 & 467 \\
\hline \multicolumn{3}{|l|}{ Serious AE } \\
\hline Total patients with $\geq 1$ serious $A E, \%(n)$ & $6.1 \%(17)$ & $5.8 \%(16)$ \\
\hline Total no of serious $A E$ & 25 & 21 \\
\hline Rate of serious AE (per $100 \mathrm{PY}$ ) & 21 & 18 \\
\hline \multicolumn{3}{|l|}{ Serious infections } \\
\hline $\begin{array}{l}\text { Total patients with } \geq 1 \text { serious infections, } \\
\% \text { (n) }\end{array}$ & $2.2 \%(6)$ & $2.2 \%(6)$ \\
\hline Total no of serious infections & 7 & 7 \\
\hline Rate of serious infections (per $100 \mathrm{PY}$ ) & 6 & 6 \\
\hline Total no of deaths & $1 \dagger$ & 2 \\
\hline ALT elevations, \% ( $n \ddagger)$ & $N \S=244$ & $N \S=243$ \\
\hline$>$ ULN-1.5 $\times$ ULN & $25.8 \%(63)$ & $16.5 \%(40)$ \\
\hline$>1.5-3 \times$ ULN & $15.2 \%(37)$ & $9.9 \%(24)$ \\
\hline$>3-5 \times$ ULN & $5.7 \%(14)$ & $0.8 \%(2)$ \\
\hline$>5 \times$ ULN & $2.0 \%(5)$ & $0.4 \%(1)$ \\
\hline AST elevations, \% (n‡) & $\mathrm{N} \S=257$ & $\mathrm{~N} \S=250$ \\
\hline$>$ ULN-1.5 × ULN & $21.8 \%(56)$ & $14.8 \%(37)$ \\
\hline$>1.5-3 \times$ ULN & $10.5 \%(27)$ & $4.0 \%(10)$ \\
\hline$>3-5 \times$ ULN & $1.9 \%(5)$ & $0.4 \%(1)$ \\
\hline$>5 \times$ ULN & $0.0 \%(0)$ & $0.0 \%(0)$ \\
\hline
\end{tabular}

${ }^{*}$ Cumulative incidences weeks 1-24, excluding patients with elevations at baseline. Data including non-fasting samples.

†An additional patient in this group had an adverse event leading to death but died only after the 24-week cut-off.

¥Number of patient with a normal baseline and the highest value in the first 24 weeks within the indicated range. ULN=55 U/I for ALT and $40 \mathrm{U} / \mathrm{I}$ for AST.

$\S$ Number of patients with normal value at baseline.

$A E$, adverse event; ALT, alanine aminotransferase (glutamate pyruvate transaminase); $A S T$, aspartate aminotransferase (glutamate oxaloacetate transaminase); MTX, methotrexate;

PBO, placebo; PY, patient years; TCZ, tocilizumab; ULN, upper limit of normal.

rejecting the clinical study hypothesis. The study was powered to detect a $12.5 \%$ difference in DAS28-ESR remission rates at week 24 between the two arms, which is the minimum difference that the scientific steering committee considered to be clinically meaningful. This choice was supported by CHARISMA, a phase II dose-finding study, which indicated that combination therapy of tocilizumab with methotrexate might be superior to monotherapy (eg, DAS28 remission at week 16 achieved by $34 \%$ of combination therapy and $17 \%$ of monotherapy patients). ${ }^{13}$ Based on the number of patients actually randomly assigned and the observed remission rate in the tocilizumab plus placebo arm the study was factually able to detect a difference of approximately $11 \%$ between the two strategies and as such had adequate discriminative power for a relevant treatment difference. The patients were selected based on the established definition of RA and their baseline characteristics were in concordance with earlier studies with tocilizumab or other biological agents, and were well balanced between the treatment arms. The only notable exception was baseline GSS, which showed more advanced structural damage in the switch group.

The doses of both methotrexate/placebo and tocilizumab were in line with approved labels. Furthermore, the methotrexate/placebo doses were similar between trial arms (table 1) and consistent with previous clinical trials evaluating combination methotrexate plus biological agent treatment ${ }^{16-20}$ as well as with commonly prescribed methotrexate doses in clinical practice. ${ }^{21-23}$ However, methotrexate/placebo doses were lower than in some current recommendations for the optimisation of methotrexate. ${ }^{24} 25$

In the absence of any methodological bias that could have artificially reduced the difference in efficacy between the two arms, we conclude that the answer to the question asked in the study 'Is the add-on strategy superior to the switch to monotherapy strategy?' is 'no' so that one could suggest that both strategies provide a similar benefit in terms of clinical and structural outcomes.

Safety outcomes were similar in the two arms, except for a numerically higher rate of increased transaminase levels with the add-on strategy. Overall, the incidence of events such as serious $\mathrm{AE}$, serious infections and discontinuations was similar between the add-on and switch strategies, consistent with previous tocilizumab studies.

The data observed in this study suggest that tocilizumab monotherapy may be a valuable treatment strategy for certain RA patients requiring biological agents. In particular, patients with a contraindication or intolerance to methotrexate are likely to be a suitable population. Further confirmation of these data is required, including through the longer term observation of the patients recruited for this study as well as additional studies in other patient sets including the evaluation in daily practice via registries.

Contributors MD, KK, PPT, PGC, EMM, GS, CB and TWJH designed the study and analysed and interpreted the data. MD, TS, PPT, PGC, EMM, GS, HA, FNS, AH and TWJH along with other investigators were involved in generating the data at their clinical research sites. All authors were involved in writing the manuscript and approved it.

Funding The ACT-RAY study was funded by F Hoffmann-La Roche Ltd, Basel, Switzerland (Roche). Roche was involved in developing the study design, in the analysis and interpretation of the data, in the writing of the report, and in the decision to submit the paper for publication, through Roche employees, contractors and funding of third party support such as contract research organisations. All these activities happened in close collaboration with the external members of the scientific steering committee.

Competing interests MD has participated in symposia and advisory boards organised by Roche and received consulting fees and his department has received research grants from Roche for conducting clinical trials and/or clinical epidemiological studies. KK is an employee of F Hoffmann-La Roche. TS received consulting fees and research grants from Roche. PPT's department has received Roche funds as grants and consulting fees or honorarium. PPT is an employee of and has stock/stock options for GlaxoSmithKline. PGC has received research grants from Centocor Inc and Roche and has been a speaker for Astra Zeneca, Bioberica, Bristol-Myers Squibb, Centocor Inc, Merck Pharmaceuticals, Novartis Pharmaceutical Corporation, Pfizer and Roche. EMM has received consulting fees and been an educational lecturer for Abbott Immunology, Roche MSD, Pfizer and UCB. GS received consulting fees from Roche. FNS has consulted for Pfizer, UCB, Abbott and Roche and has been a speaker for Roche, Pfizer, Abbott and MSD, and his department has received research grants from Roche, Pfizer and Abbott. AH has no competing interests to report. CB is a contractor of $\mathrm{F}$ Hoffmann-La Roche. TWJH has received consulting fees and has been a speaker for Abbott Immunology, Axis Shield Diagnostics, Biotest AG, Bristol-Myers Squibb, Crescendo Bioscience, Roche, Novartis Pharmaceuticals, Schering-Plough, UCB and Wyeth-Pfizer.

Ethics approval The study was approved by the appropriate institutional review boards/ethics committees.

\section{Patient consent Obtained.}

Provenance and peer review Not commissioned; externally peer reviewed.

Open Access This is an Open Access article distributed in accordance with the Creative Commons Attribution Non Commercial (CC BY-NC 3.0) license, which permits others to distribute, remix, adapt, build upon this work non-commercially, and license their derivative works on different terms, provided the original work is properly cited and the use is non-commercial. See: http://creativecommons.org/ licenses/by-nc/3.0/ 


\section{REFERENCES}

1. Smolen JS, Aletaha D, Bijlsma JW, et al. Treating rheumatoid arthritis to target: recommendations of an international task force. Ann Rheum Dis 2010;69:631-7.

2. Bykerk V. Unmet needs in rheumatoid arthritis. J Rheumato/ Supp/ 2009;82:42-6.

3. Genant HK, Jiang Y, Peterfy C, et al. Assessment of rheumatoid arthritis using a modified scoring method on digitized and original radiographs. Arthritis Rheum 1998;41:1583-90.

4. Smolen JS, Landewé R, Breedveld FC, et al. EULAR recommendations for the management of rheumatoid arthritis with synthetic and biological disease-modifying antirheumatic drugs. Ann Rheum Dis 2010;69:964-75.

5. Listing J, Strangfeld A, Rau R, et al. Clinical and functional remission: even though biologics are superior to conventional DMARDs overall success rates remain low - results from RABBIT, the German biologics register. Arthritis Res Ther 2006; 8:R66.

6. Soliman MM, Ashcroft DM, Watson KD, et al. Impact of concomitant use of DMARDs on the persistence with anti-TNF therapies in patients with rheumatoid arthritis: results from the British Society for Rheumatology Biologics Register. Ann Rheum Dis 2011;70:583-9.

7. Feely MG, O'Dell JR. Update on the use of conventional disease-modifying antirheumatic drugs in the management of rheumatoid arthritis. Curr Opin Rheumatol 2010;22:316-20.

8. Sebba A. Tocilizumab: the first interleukin-6-receptor inhibitor. Am J Health Syst Pharm 2008;65:1413-18.

9. Smolen JS, Beaulieu A, Rubbert-Roth A, et al. Effect of interleukin-6 receptor inhibition with tocilizumab in patients with rheumatoid arthritis (OPTION study): a double-blind, placebo-controlled, randomised trial. Lancet 2008;371:987-97.

10. Watanabe C, Sumioka M, Hiramoto T, et al. Magnifying colonoscopy used to predict disease relapse in patients with quiescent ulcerative colitis. Inflamm Bowel Dis 2009;15:1663-9.

11. Aletaha D, Landewe R, Karonitsch $T$, et al. Reporting disease activity in clinical trials of patients with rheumatoid arthritis: EULAR/ACR collaborative recommendations. Ann Rheum Dis 2008:67:1360-4.

12. Jones G, Sebba A, Gu J, et al. Comparison of tocilizumab monotherapy versus methotrexate monotherapy in patients with moderate to severe rheumatoid arthritis: the AMBITION study. Ann Rheum Dis 2010;69:88-96.

13. Maini RN, Taylor PC, Szechinski J, et al. Double-blind randomized controlled clinical trial of the interleukin-6 receptor antagonist, tocilizumab, in European patients with rheumatoid arthritis who had an incomplete response to methotrexate. Arthritis Rheum 2006; 54:2817-29.

14. Bruynesteyn K, Boers M, Kostense P, et al. Deciding on progression of joint damage in paired films of individual patients: smallest detectable difference or change. Ann Rheum Dis 2005;64:179-82.
15. Felson DT, Smolen JS, Wells G, et al. American College of Rheumatology/European League against Rheumatism provisional definition of remission in rheumatoid arthritis for clinical trials. Ann Rheum Dis 2011;70:404-13.

16. Keystone EC, Genovese MC, Klareskog L, et al. Golimumab, a human antibody to tumour necrosis factor \{alpha\} given by monthly subcutaneous injections, in active rheumatoid arthritis despite methotrexate therapy: the GO-FORWARD Study. Ann Rheum Dis 2009;68:789-96.

17. Breedveld FC, Weisman MH, Kavanaugh AF, et al. The PREMIER study: a multicenter, randomized, double-blind clinical trial of combination therapy with adalimumab plus methotrexate versus methotrexate alone or adalimumab alone in patients with early, aggressive rheumatoid arthritis who had not had previous methotrexate treatment. Arthritis Rheum 2006;54:26-37.

18. Keystone EC, Kavanaugh AF, Sharp JT, et al. Radiographic, clinical, and functional outcomes of treatment with adalimumab (a human anti-tumor necrosis factor monoclonal antibody) in patients with active rheumatoid arthritis receiving concomitant methotrexate therapy: a randomized, placebo-controlled, 52-week trial. Arthritis Rheum 2004;50:1400-11.

19. Maini R, St Clair EW, Breedveld F, et al. Infliximab (chimeric anti-tumour necrosis factor alpha monoclonal antibody) versus placebo in rheumatoid arthritis patients receiving concomitant methotrexate: a randomised phase III trial. ATTRACT Study Group. Lancet 1999;354:1932-9.

20. Weinblatt ME, Keystone EC, Furst DE, et al. Adalimumab, a fully human anti-tumor necrosis factor alpha monoclonal antibody, for the treatment of rheumatoid arthritis in patients taking concomitant methotrexate: the ARMADA trial. Arthritis Rheum 2003;48:35-45

21. Salliot C, van der Heijde D. Long-term safety of methotrexate monotherapy in patients with rheumatoid arthritis: a systematic literature research. Ann Rheum Dis 2009:68:1100-4.

22. Aletaha D, Stamm T, Kapral T, et al. Survival and effectiveness of leflunomide compared with methotrexate and sulfasalazine in rheumatoid arthritis: a matched observational study. Ann Rheum Dis 2003;62:944-51.

23. Mainman H, McClaren E, Heycock C, et al. When should we use parenteral methotrexate? Clin Rheumatol 2010;29:1093-8.

24. Visser K, Katchamart W, Loza E, et al. Multinational evidence-based recommendations for the use of methotrexate in rheumatic disorders with a focus on rheumatoid arthritis: integrating systematic literature research and expert opinion of a broad international panel of rheumatologists in the $3 \mathrm{E}$ Initiative. Ann Rheum Dis 2009;68:1086-93.

25. Pavy S, Constantin A, Pham T, et al. Methotrexate therapy for rheumatoid arthritis: clinical practice guidelines based on published evidence and expert opinion. Joint Bone Spine 2006;73:388-95 